\title{
Gastrointestinale Blutung
}

\author{
Ursula Wehrmann $^{\mathrm{a}}$ Georg Kähler ${ }^{\mathrm{b}}$ Jürgen Hochberger ${ }^{\mathrm{c}}$ \\ ${ }^{a}$ Klinik und Poliklinik für Viszeral-, Thorax- und Gefäßchirurgie, Universitätsklinikum Carl Gustav Carus, Technische Universität Dresden,

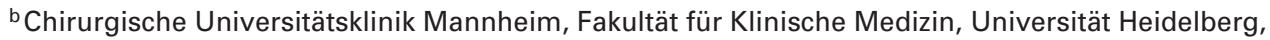 \\ ${ }^{\mathrm{c}}$ Medizinische Klinik III, St. Bernward Krankenhaus, Hildesheim, Deutschland
}

Die akute gastrointestinale Blutung stellt für den Patienten eine lebensbedrohliche Situation dar. Die Letalität der oberen nichtvarikösen gastrointestinalen Blutung, die im Wesentlichen durch das Alter des Patienten, die Komorbidität, die Blutungsaktivität und die Blutungsintensität beeinflusst wird, liegt trotz Weiterentwicklung der diagnostischen und therapeutischen Endoskopie und der intensivmedizinischen Techniken nach wie vor bei $6-14 \%$.

Nahezu 90\% der Blutungsquellen sind im oberen Gastrointestinaltrakt lokalisiert. Dabei sind peptische Läsionen (50-60\%) am häufigsten, gefolgt von Ösophagus- und Magenfundusvarizen (10-30\%).

10-20\% der gastrointestinalen Blutungen haben ihren Ursprung jenseits des Treitz'schen Bandes und werden definitionsgemäß als untere gastrointestinale Blutung klassifiziert. 80-85\% der unteren gastrointestinalen Blutungen sistieren spontan und gehen mit einer im Vergleich zur oberen gastrointestinalen Blutung niedrigeren Letalität einher. Während bei jüngeren Patienten vorrangig die Hämorrhoidalblutung als Ursache der unteren Gastrointestinalblutung zu finden ist, kommt bei älteren Patienten die Divertikelblutung am häufigsten vor. 5-10\% der Blutungsquellen bei der unteren gastrointestinalen Blutung sind im Dünndarm lokalisiert und stellen eine besondere Herausforderung an das diagnostische und therapeutische Management dar. Bei Dünndarmblutungen wird heute auch von einer mittleren gastrointestinalen Blutung gesprochen.

Die primäre Diagnostik und Therapie der gastrointestinalen Blutung ist die Domäne der Endoskopie. Der Erfolg der primären endoskopischen Blutstillung bei der akuten gastrointestinalen Blutung wird dabei nicht zuletzt von der Erfahrung des Endoskopikers und des Endoskopieassistenzpersonals bestimmt. Neben der Lokalisation und diagnostischen Einordnung der Blutungsquelle sowie der definitiven Blutstillung ist die Information zur Prognose mit Einschät- zung des Risikos der Rezidivblutung ein wichtiger Zielparameter der Notfallendoskopie. Die frühzeitige Diagnostik und Therapie der gastrointestinalen Blutung erfordert eine jederzeit verfügbare Notfallendoskopie mit der erforderlichen personellen Kompetenz. Dabei ist es nicht relevant, ob ein kompetenter Chirurg oder Gastroenterologe agiert. Entscheidend für eine erfolgreiche Therapie der gastrointestinalen Blutung mit dem Ziel der Senkung der Letalität ist die interdisziplinäre Zusammenarbeit von Internisten, Viszeralchirurgen, interventionell tätigen Radiologen und Intensivtherapeuten.

Daher begrüßen wir es als Gastherausgeber außerordentlich, dass wir mit der Gestaltung des vorliegenden Hefts der CHIRURGISCHE GASTROENTEROLOGIE INTERDISZIPLINÄR die Gelegenheit erhalten haben, die interdisziplinären Aspekte, die in besonderem Maße für die Diagnostik und Therapie der gastrointestinalen Blutung von Bedeutung sind, zu verdeutlichen. Neben bereits bewährten effektiven Blutstillungsmaßnahmen werden neue Entwicklungen aufgezeigt und bewertet. Dabei haben die Fortschritte in der Therapie von Magen- und Duodenalulzera durch den Einsatz von Protonenpumpeninhibitoren, durch die Helicobacter-Eradikation sowie durch die Weiterentwicklung endoskopischer Techniken die operative Therapie der Ulkusblutung in ihrer Häufigkeit zurückgedrängt. Erste Berichte zeigen, dass für die Therapie der 5-10\% endoskopisch nicht beherrschbaren Blutungen zukünftig radiologisch interventionelle Embolisationstechniken von Bedeutung sein könnten. Inwieweit dadurch eine weitere Reduzierung der Operationsfrequenz möglich wird, müssen Studien belegen.

Hämoclips sind heute bereits eine effektive Bereicherung des therapeutischen Spektrums der endoskopischen Blutstillung sowohl im oberen als auch im unteren Gastrointestinaltrakt. In einem Beitrag dieses Hefts werden die auf dem Markt befindlichen Clip-Systeme vorgestellt und dem Anwender an

\begin{tabular}{ll}
\hline KARGER & ( ) 2005 S. Karger GmbH, Freiburg \\
Fax +49 7614520714 & Accessible online at: \\
$\begin{array}{l}\text { E-mail Information@Karger.de } \\
\text { www.karger.com }\end{array}$ & www.karger.com/cga
\end{tabular}

Dr.med. U.Wehrmann

Klinik und Poliklinik für Viszeral-, Thorax- und Gefäßchirurgie

Universitätsklinikum Carl Gustav Carus, Technische Universität Dresden

Fetscherstraße 7401307 Dresden, Deutschland

Tel. +49351458-2075, Fax -5871

E-mail Ursula.wehrmann@uniklinikum-dresden.de 
Hand experimenteller und klinischer Daten wertvolle Hinweise für die Praxis gegeben.

Die medikamentöse und endoskopische Therapie der varikösen Blutung wird in ihrer Komplexität dargestellt. Welche Rolle möglicherweise zukünftig die TIPS in der Prävention der varikösen Blutung spielen wird, diskutiert ein weiterer Beitrag.

Mit der Doppelballonenteroskopie und der Kapselendoskopie wurde das diagnostische und im Fall der Doppelballonenteroskopie auch das therapeutische Spektrum für die seltenen, aber dafür häufig komplizierten Dünndarmblutungen erweitert. Mit der Entwicklung der Kapselendoskopie werden mögliche neue Wege für eine minimal invasive Diagnostik des Dünndarms aufgezeigt. Fehlende Interventionsmöglichkeiten schränken den kostenintensiven Einsatz jedoch ein. Für die akute diagnostisch und therapeutisch nicht zugängliche, persistierende oder auch rezidivierende intestinale Blutung bleibt vorerst die Laparotomie mit intraoperativer Endoskopie das Standardverfahren. In Einzelfällen kann die Laparoskopie mit der intraoperativen Endoskopie kombiniert werden. 